\title{
Linkage Analysis of Genomic Regions Contributing to the Expression of Type 1 Diabetes Microvascular Complications and Interaction with HLA
}

\author{
Ettie M. Lipner, ${ }^{1,2}$ Yaron Tomer, ${ }^{3}$ Janelle A. Noble, ${ }^{4}$ Maria C. Monti, ${ }^{5}$ John T. Lonsdale, ${ }^{6}$ \\ Barbara Corso, ${ }^{5}$ and David A. Greenberg ${ }^{7,8}$ \\ ${ }^{1}$ Integrated Center for Genes, Environment and Health, National Jewish Health, Denver, CO 80206, USA \\ ${ }^{2}$ Department of Pharmacology, University of Colorado Denver School of Medicine, Aurora, CO 80045, USA \\ ${ }^{3}$ Department of Medicine, Mount Sinai Medical Center, New York, NY 10013, USA \\ ${ }^{4}$ Children's Hospital Oakland Research Institute, Oakland, CA 94702, USA \\ ${ }^{5}$ National Research Council, Neuroscience Institute, 35128 Padova, Italy \\ ${ }^{6}$ National Disease Research Interchange, Philadelphia, PA 19103, USA \\ ${ }^{7}$ Battelle Center for Mathematical Medicine, Nationwide Children's Hospital, Columbus, OH 43215, USA \\ ${ }^{8}$ Department of Pediatrics, Wexner Medical Center, Ohio State University, Columbus, OH 43205, USA
}

Correspondence should be addressed to David A. Greenberg; david.greenberg@nationwidechildrens.org

Received 5 November 2014; Revised 29 January 2015; Accepted 8 February 2015

Academic Editor: Gareth McKay

Copyright (C) 2015 Ettie M. Lipner et al. This is an open access article distributed under the Creative Commons Attribution License, which permits unrestricted use, distribution, and reproduction in any medium, provided the original work is properly cited.

\begin{abstract}
We conducted linkage analysis to follow up earlier work on microvascular complications of type 1 diabetes (T1D). We analyzed 415 families (2,008 individuals) previously genotyped for 402 SNP markers spanning chromosome 6 . We did linkage analysis for the phenotypes of retinopathy and nephropathy. For retinopathy, two linkage peaks were mapped: one located at the HLA region and another novel locus telomeric to HLA. For nephropathy, a linkage peak centromeric to HLA was mapped, but the linkage peak telomeric to HLA seen in retinopathy was absent. Because of the strong association of T1D with DRB1 03:01 and DRB1 04:01, we stratified our analyses based on families whose probands were positive for DRB1 03:01 or DRB1 04:01. When analyzing the DRB1 $^{*}$ 03:01-positive retinopathy families, in addition to the novel telomeric locus, one centromeric to HLA was identified at the same location as the nephropathy peak. When we stratified on DRB1 $1^{*}$ 4:01-positive families, the HLA telomeric peak strengthened but the centromeric peak disappeared. Our findings showed that HLA and non-HLA loci on chromosome 6 are involved in T1D complications' expression. While the HLA region is a major contributor to the expression of T1D, our results suggest an interaction between specific HLA alleles and other loci that influence complications' expression.
\end{abstract}

\section{Introduction}

Retinopathy, nephropathy, and neuropathy are chronic microvascular complications responsible for much of the morbidity and mortality in type 1 diabetes (T1D). Evidence for familiarity in complications has been clearly demonstrated, suggesting a genetic contribution to these phenotypes [1-4]. Although numerous linkage and association studies have focused on identifying T1D susceptibility loci, there has been little analysis of genetic influences on complications.
In the few linkage analyses that focused on identifying TiDrelated complications of susceptibility loci, only nephropathy has been investigated [5-9]. To our knowledge, there have been no linkage studies aiming at investigating the influence of the HLA region on the expression of complications.

Therefore, the aim of our study was to use the robust method of linkage analysis in a large well-characterized cohort of T1D families to identify gene-loci that predispose to type 1 diabetic complications. We focused our genome analysis on chromosome 6 to follow up our previous work 
showing the importance of loci on chromosome 6 to the genetic predisposition of T1D complications [10].

\section{Methods}

2.1. Family Recruitment and Data Collection. Families were ascertained through the presence of at least one family member with type 1 diabetes. A questionnaire was given to the proband or parents as well as to additional family members. The questionnaire included demographic, medical, genealogical, and familial information about T1D as well as complications. More details can be found in Lipner et al. [10]. Other information to assure the accuracy of participants' disease status is discussed below.

2.2. HBDI Data. Our dataset included 415 families $(2,008$ individuals) with T1D cases diagnosed before age 30 (Tables 1 and 2). Female percentage was 49\%. 239 individuals in 159 families had at least 1 microvascular complication: 219 individuals had retinopathy, 87 had nephropathy, and 76 had neuropathy. Some subjects had more than one complication.

2.3. Assessment of Diabetes and Diabetic Complications. The accuracy of the self-reported information about complications was evaluated by the following.

(1) Including extra questions about complicationsrelated conditions in the questionnaire. Reports of macular edema, vitrectomy, or complete or partial blindness were considered an indicator of retinopathy; reports of end-stage renal failure, kidney failure, or repeated high urinary albumin levels were considered an indicator of nephropathy. In cases of inconsistencies (e.g., report of macular edema but not retinopathy), further investigations were carried out through phone interviews. In order to avoid ambiguity, only the most obvious or severe cases of retinopathy or nephropathy were classified as "affected."

(2) Data available from follow-up were used to confirm or update the presence/absence and progression of complications.

(3) 179 patients had medical records available allowing us to verify phenotype according to American Diabetes Association guidelines [11-14].

(4) Information indicating absence of a complication in a family member with T1D was considered reliable only if the subject was without that complication for at least 15 years after type 1 diabetes onset.

2.4. Assessment of Self-Reported Diabetic Complications. The accuracy of self-reported information was assessed in three ways. (1) Additional questions were included in questionnaires given to both patients and family members. (2) Followup telephone interviews were carried out by HBDI staff if the questionnaire was unclear. (3) Medical records were assessed on T1D patients that submitted medical records with
TABLE 1: Number of families with affected (T1D + complications)unaffected (T1D only) members.

\begin{tabular}{lc}
\hline Affected-unaffected family members & $N_{\text {families }}(\%)$ \\
\hline 1 affected-1 unaffected & $68(16)$ \\
2 affected-0 unaffected & $50(12)$ \\
0 affected-2 unaffected & $210(51)$ \\
Other & $87(21)$ \\
\hline Total & $415(100)$ \\
\hline
\end{tabular}

TABle 2: Prevalence of clinical characteristics among 415 T1D families.

\begin{tabular}{lc}
\hline Clinical characteristic & Number (\%) of individuals \\
\hline Total & $2,008(100.0)$ \\
T1D + microvascular complications & $239(11.9)$ \\
T1D + retinopathy & $219(91.6)$ \\
T1D + nephropathy & $87(36.4)$ \\
T1D + neuropathy & $76(31.8)$ \\
T1D only & $629(31.3)$ \\
No T1D & $1140(56.8)$ \\
\hline
\end{tabular}

the questionnaire (179 (2.3\%)). (4) Follow-up questionnaires went to a subset of families for updated information about the development of complications, new cases of diabetes, and other related medical history. Twenty-three percent of the type 1 diabetics in the HBDI database responded with followup data and $10 \%$ of subjects included medical records with the questionnaire. On-going validation at HBDI has shown that questionnaire answers accurately reflect physician diagnosis in the medical records [1]. Thus, the severity of reported symptoms, corroboration of accuracy using patients' medical records, and follow-up contact with a sample of patients and families assure phenotype accuracy.

Since the majority of patients' diagnoses are self-reported, T2D may have occasionally been misclassified as T1D. The presence of autoantibodies would confirm an autoimmune response. Autoantibody markers from a random sample of T1D study subjects $(n=76)$ characterized study sample homogeneity. Only 5\% of T1D-classified patients in this subsample tested negative for autoantibodies. Also, the absence of autoantibodies is not proof of misdiagnosis. 3.5\%-10\% of T1D patients are autoantibody-negative $[15,16]$. Thus, misdiagnosis of T2D as T1D is unlikely to have affected our results.

Reliability of self-report questionnaires: self-reports of diabetes have demonstrated excellent agreement with the use of medical records [17]. Further, other studies have shown that self-reporting of diabetes tends to be more accurate than other chronic disease self-reports $[18,19]$. We did not identify any studies comparing the use of medical records with selfreport of diabetic microvascular complications.

Thus, if any T1D families were actually T2D, or if some patients with complications were misdiagnosed as complications-free, it is unlikely to have introduced bias into our results for two major reasons. (1) Misdiagnosing an 
affected person as "unaffected" decreases linkage evidence but does not lead to false linkage evidence [20]; it introduces reduced penetrance, which the analysis takes account of through the penetrance parameter. (2) Classifying a truly unaffected person as "affected" has the effect of severely reducing the evidence for linkage but can be taken into account via the HLOD parameter. Strong evidence of linkage, with high LOD and HLOD scores, does not eliminate the possibility of misdiagnosis or heterogeneity but finding false evidence of linkage because of heterogeneity or misdiagnosis is highly unlikely, since any misdiagnosis degrades the linkage signal.

2.5. Genotyping. The Center for Inherited Disease Research (CIDR) at the National Human Genome Research Institute did the genotyping. Average marker spacing was $0.58 \mathrm{cM}$. We restricted our analyses to the 402 marker SNPs on chromosome 6.

2.6. Phenotype Definitions. "Affected" phenotypes were (1) the presence of any microvascular complication, (2) the presence of retinopathy, and (3) the presence of nephropathy. Each phenotype was analyzed separately. The neuropathy phenotype yielded too little linkage information and no further analyses were done using that phenotype. T1D patients without complications were classified as "unaffected." Individuals without T1D were excluded from these analyses (except parents). Families had at least one "affected" and one "unaffected" family member, or at least two affected members (e.g., at least two siblings with T1D, at least one of whom had complications).

2.7. Linkage Analysis. Multipoint LOD ("logarithm of odds") scores and heterogeneity LOD scores (HLOD scores) were calculated using the GeneHunter program [21]. The HLOD reflects the evidence for linkage taking into account possible heterogeneity within the dataset; that is, only a proportion of families in the dataset are linked to the marker. We assumed both dominant and recessive modes of inheritance [22]. A dominant gene frequency of 0.1 and a recessive gene frequency of 0.2 were assumed. Preliminary analyses assuming three levels of penetrance $(90 \%, 50 \%$, and $25 \%)$ and a dominant and recessive mode of inheritance showed that a penetrance of $25 \%$ and recessive inheritance yielded the highest LOD scores [20, 21, 23, 24]. Subsequently, for all reported analyses, we assumed a recessive mode of inheritance and $25 \%$ penetrance. In all calculations, if assuming a recessive mode of inheritance led to positive LOD scores, so did assuming a dominant inheritance, indicating evidence in favor of linkage irrespective of assumed mode of inheritance. Almost entirely, the LOD score assuming a recessive inheritance model was notably higher than dominant, so the LOD and HLOD assuming recessive inheritance are the scores we report.

We performed preliminary analyses on the phenotype "any complication" but our subsequent analysis classified only subjects with retinopathy (RET) as "affected" and, separately, only subjects with nephropathy (NEPH) as "affected."
2.8. Stratification on the Presence of T1D High-Risk HLA Alleles in the Proband. We previously showed [10] that the DRB1 ${ }^{*}$ 03:01 allele provided a protective effect against retinopathy. Therefore, we explored the influence of DRB1 $^{*} 03: 01$ or DRB1 ${ }^{*} 04: 01$ on the linkage evidence in subsets of families grouped by the presence of DRB1 ${ }^{*}$ 03:01 or DRB1 ${ }^{*}$ 4:01 in the proband. Our aim in stratifying was to identify possible gene-gene interaction between the novel loci we identified and these HLA alleles, since we had previously identified the alleles' differential effect on complications risk [10]. We also carried out "pure" DRB1 03:01 or DRB1*04:01 analyses in which the probands of the selected families carried only the DRB1 $03: 01 / \mathrm{X}(\mathrm{X} \neq 04: 01)$ or $\mathrm{DRB1}^{*} 04: 01 / \mathrm{X}$ $(X \neq 03: 01)$ genotype. Changes in the LOD score profiles in these different subgroups can reflect interaction of that allele with loci linked to the phenotype [25].

\section{Results}

3.1. Linkage Analysis with "Any Complication" as the Phenotype, (Figure 1(a)). With the affected phenotype defined as "presence of any complication," a large linkage peak emerged centered in the HLA region (50-52 cM); the LOD and HLOD scores at $52 \mathrm{cM}$ (HLA region location) were 4.0 and 5.3, respectively. Two separate, novel loci for complications were located outside the HLA region (Table 4, Figure 1(a)), one telomeric $(42 \mathrm{cM})$ and one centromeric $(64 \mathrm{cM})$ to the HLA region. At an assumed penetrance of 0.25 , the LOD was 2.6 at the $42 \mathrm{cM}$ peak; the HLOD was 4.4. The LOD score at the centromeric region $(64 \mathrm{cM})$ was negative, but the HLOD score was 2.6 (Table 4, Figure 1(a)), suggesting linkage in only a subset of the families.

\subsection{Linkage Analysis with Retinopathy as the Phenotype} (RET), (Figure 1(b)). RET was the most common complication found in our dataset. We saw only minor differences between the ANY COMPLICATION and RET analyses. The maximum scores at the $42 \mathrm{cM}$ peak for RET were LOD = 3.6 and HLOD $=5.0$ (for ANY COMPLICATION, the scores were $\mathrm{LOD}=2.6$ and HLOD $=4.4$ (compare Figures $1(\mathrm{a})$ and 1(b), Table 4)). At the HLA locus, the scores for RET were $\mathrm{LOD}=3.6$ and $\mathrm{HLOD}=5.0$, and for ANY COMPLICATION, they were $\mathrm{LOD}=4.0$ and $\mathrm{HLOD}=5.3$. The HLOD scores at the broad peak around $64 \mathrm{cM}$ were 2.2 for RET and 2.6 for ANY COMPLICATION. It is noteworthy that the LOD scores at the $42 \mathrm{cM}$ peak increased significantly when including only RET as "affected" compared with ANY COMPLICATION, despite the drop in sample size, that is, excluding nephropathy and neuropathy cases. This suggests that the $42 \mathrm{cM}$ peak does not contribute to the expression of nephropathy or neuropathy.

3.3. Linkage Analysis with Nephropathy as the Phenotype (NEPH), (Figure 1(c)). The linkage results in the $45 \mathrm{NEPH}$ families show 2 peaks: the first peak occurs over the HLA region at $52 \mathrm{cM}(\mathrm{LOD}=1.3$ and HLOD $=1.4($ Figure $1(\mathrm{c})))$. The second peak is located at the same position $(64 \mathrm{cM})$ as the centromeric peak seen in the ANY COMPLICATION 


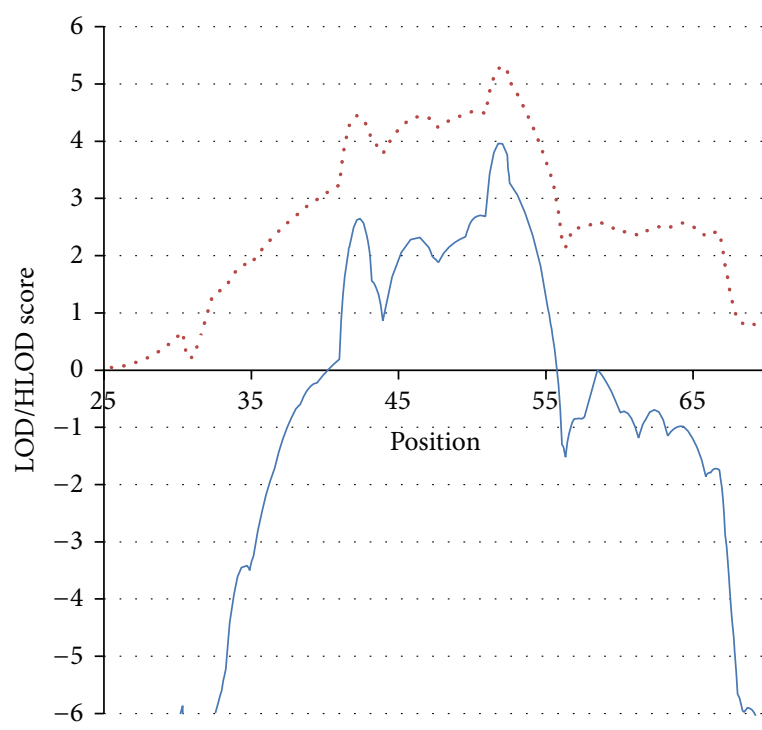

(a)

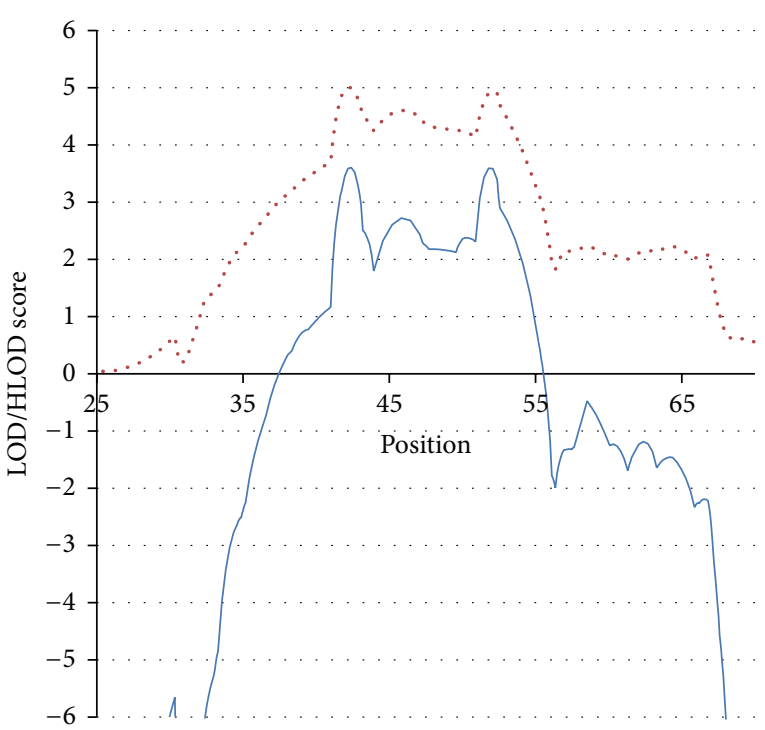

(b)

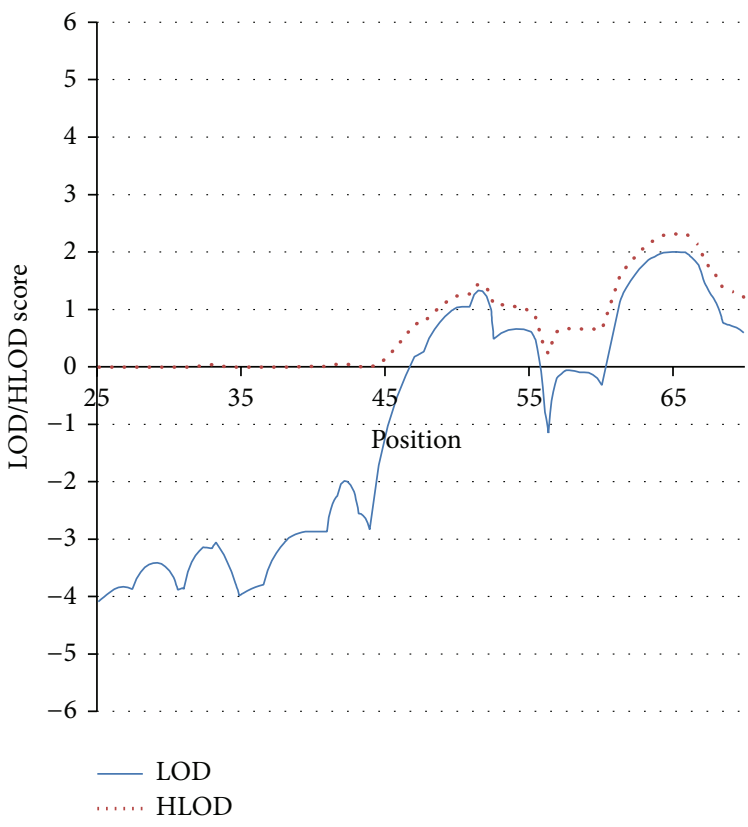

(c)

FIGURE 1: (a) Linkage analysis with "any complication" as the phenotype. (b) Linkage analysis with retinopathy as the phenotype. (c) Linkage analysis with nephropathy as the phenotype.

and RET analyses. There is evidence against linkage with the NEPH phenotype at the $42 \mathrm{cM}$ locus that showed strong linkage evidence for RET and ANY COMPLICATION. Furthermore, with the NEPH phenotype, the $64 \mathrm{cM}$ peak shows virtually no evidence of heterogeneity (similar LOD and HLOD values $(\mathrm{LOD}=2.0$ and $\mathrm{HLOD}=2.2)$, suggesting that it is a locus uniquely linked with NEPH. This suggests that the $42 \mathrm{cM}$ locus is unique to RET and does not influence NEPH.

These results reveal two novel loci that contribute to the expression of complications. These two loci appear to have differential influences on RET and NEPH: one influencing mostly NEPH $(64 \mathrm{cM})$ and the other influencing only RET
( $42 \mathrm{cM})$. We then investigated possible interaction of these loci with HLA allele.

\subsection{Stratification Analyses with Retinopathy as the Phenotype}

3.4.1. DRB1 03:01 Stratification (DRB1 $\left.1^{*} 03: 01 / X\right)$, (Figure 2(a)). We previously showed [10] that the presence of DRB1 ${ }^{*}$ 03:01 reduced the risk for retinopathy while DRB1 ${ }^{*}$ 04:01 increased the risk. Therefore, we repeated the current analysis subsetting out the RET families in which the proband (a) carried the DRB1 ${ }^{*}$ 03:01 allele, including those with the 03:01/04:01 


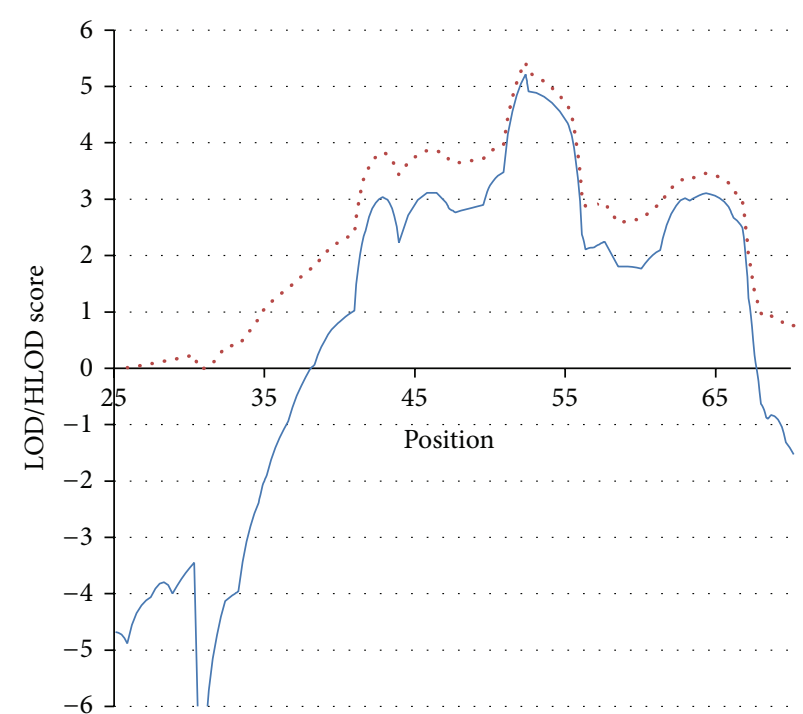

(a)

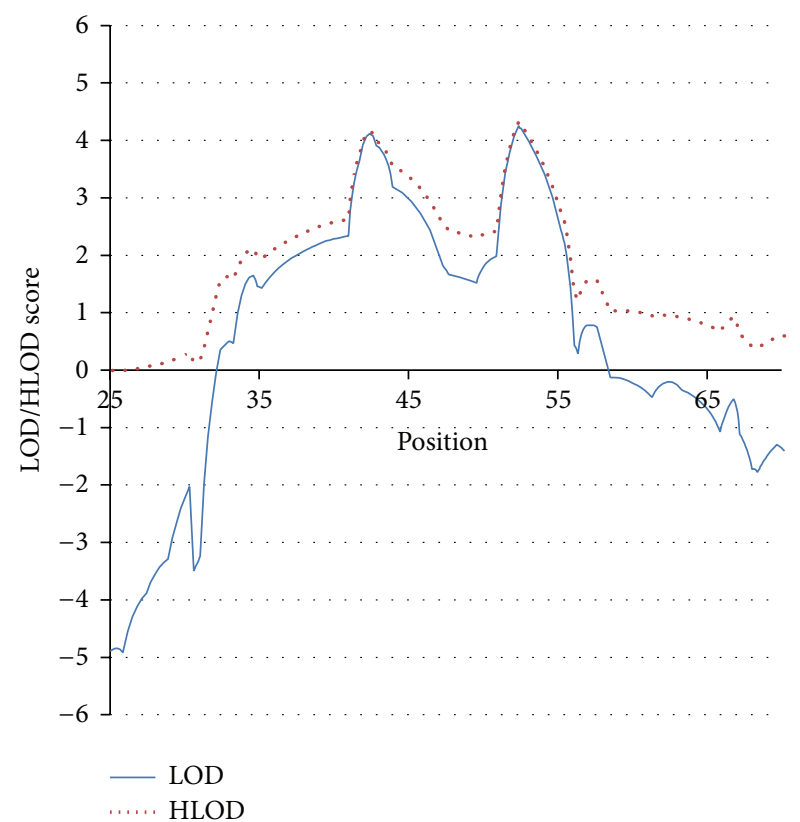

(c)

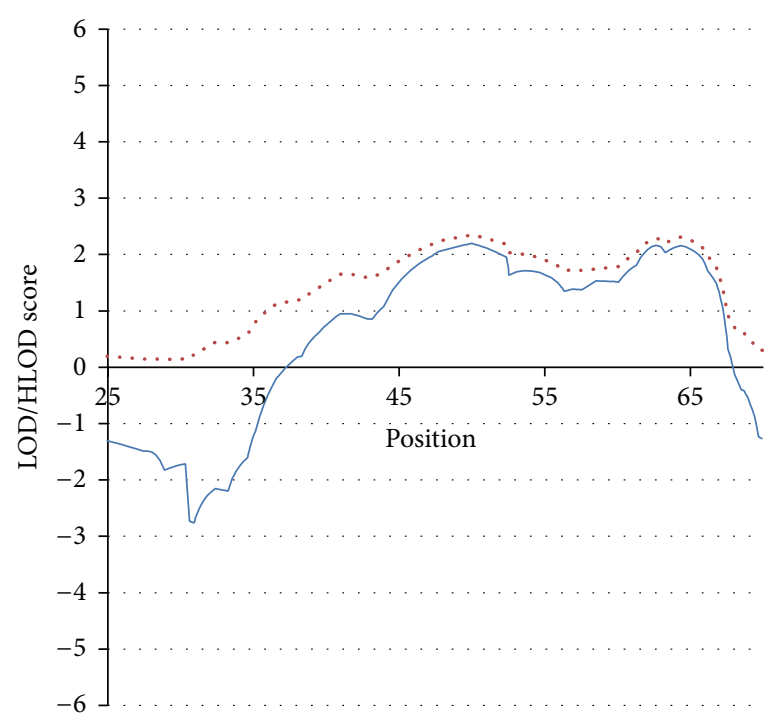

(b)

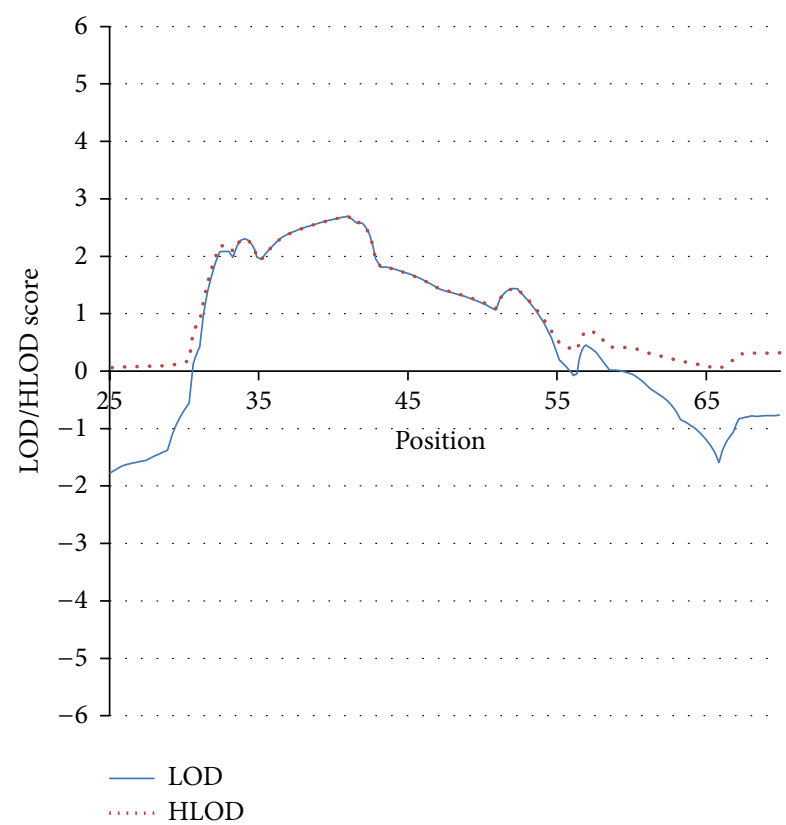

(d)

FIGURE 2: (a) Linkage analysis with retinopathy as the phenotype, stratified by families whose probands are positive for $\mathrm{DRB1} 1^{*} 03: 01 / \mathrm{X}=$ any allele. (b) Linkage analysis with retinopathy as the phenotype, stratified by families whose probands are positive for DRB1 03:01/X, where $\mathrm{X} \neq \mathrm{DRB1}^{*}$ 04:01. (c) Linkage analysis with retinopathy as the phenotype, stratified by families whose probands are positive for DRB1 $04: 01 / \mathrm{X}$. (d) Linkage analysis with retinopathy as the phenotype, stratified by families whose probands are positive for DRB1 $04: 01 / \mathrm{X}=$ any allele, where $\mathrm{X} \neq \mathrm{DRB1} 1^{*}$ 03:01.

genotype and (b) subsetting out those that carried the DRB1 03:01 allele, but excluding those with the 03:01/04:01 genotype ("pure" DRB1"03:01). We did the two analyses to disentangle the possibly opposite effects on complications of the two different HLA-DRB1 alleles seen in our previous association analysis.

At the $42 \mathrm{cM}$ locus, the unstratified RET analysis (above) had obtained a LOD $=3.6$ and an HLOD $=5.0$. The
$\mathrm{DRB1}^{*}$ 03:01 stratification analysis still showed strong evidence for linkage, but the LOD and HLOD scores decreased ( $\mathrm{LOD}=3.0$ and HLOD $=3.9)$, the decrease suggesting only that some families contributing to disease expression have been removed from the data. However, at the $64 \mathrm{cM}$ locus, there was a significant increase in linkage evidence with stratification $(\mathrm{LOD}=3.1$ and HLOD $=3.4)$ on DRB1 ${ }^{*} 03: 01$ (unstratified analysis was LOD $=-1.5$ and $\mathrm{HLOD}=2.2$ ). 
TABLE 3: LOD score summary table.

\begin{tabular}{|c|c|c|c|}
\hline \multicolumn{4}{|c|}{ LOD (HLOD) scores } \\
\hline Phenotype & $42 \mathrm{cM}$ peak & $52 \mathrm{cM}$ peak & $64 \mathrm{cM}$ peak \\
\hline Any complication & $2.6(4.4)$ & $4.0(5.3)$ & $-1(2.6)$ \\
\hline Retinopathy & $3.6(5.0)$ & $3.6(5.0)$ & $-1.5(2.2)$ \\
\hline Nephropathy & $-2.0(0.0)$ & $1.3(1.4)$ & $2.0(2.2)$ \\
\hline Retinopathy + nephropathy analyzed together & $3.2(4.8)$ & $4.0(5.2)$ & $-1.1(2.3)$ \\
\hline \multicolumn{4}{|c|}{ Stratification (retinopathy only) } \\
\hline DRB1 ${ }^{*} 03: 01$ & $3.0(3.9)$ & $5.1(5.3)$ & $3.1(3.4)$ \\
\hline $\mathrm{DRB1}^{*} 04: 01$ & $4.1(4.1)$ & $4.2(4.2)$ & $-0.5(0.9)$ \\
\hline $\begin{array}{l}\mathrm{DRB1}^{*} 03: 01 / \mathrm{X} \\
\left(\mathrm{X} \neq \mathrm{DRB1} 1^{*} 04: 01\right)\end{array}$ & $0.9(1.6)$ & $2.0(2.2)$ & $2.0(2.2)$ \\
\hline $\begin{array}{l}\mathrm{DRB1}^{*} 04: 01 / \mathrm{X} \\
\left(\mathrm{X} \neq \mathrm{DRB1}^{*} 03: 01\right)\end{array}$ & $2.5(2.5)$ & $1.4(1.4)$ & $-1.0(0.0)$ \\
\hline
\end{tabular}

Numbers in parentheses are HLODs.

TABLE 4: Numbers of families in the subgroups.

\begin{tabular}{lccc}
\hline Complication & Stratification subgroup & Number of families included & Count of people included \\
\hline Any complication & & 159 families & 1015 people \\
\hline & All families & 144 families & 928 people \\
Retinopathy & DRB1 $^{*} 03: 01$ & 61 families & 409 people \\
& DRB1 $^{*} 04: 01$ & 58 families & 336 people \\
& Pure DRB1 $^{*} 03: 01$ & 37 families & 266 people \\
Nephropathy & Pure DRB1 $^{*} 04: 01$ & 35 families & 199 people \\
\hline
\end{tabular}

This finding suggests that the $64 \mathrm{cM}$ locus may influence the expression of RET mainly in DRB1 03:01 carriers, suggesting an interaction between DRB1 ${ }^{*}$ 03:01 and the $64 \mathrm{cM}$ locus.

\subsection{2. "Pure" DRB1 03:01 Stratification (DRB1 03:01/X,} $(X \neq 04: 01)$ ), (Figure 2(b)). The above stratification analysis included $\mathrm{DRB1}^{\star}$ 03:01 positive probands with the $\mathrm{DRB1}^{*}$ 03:01/04:01 genotype. Because our previous association analysis [10] suggested opposite effects of DRB1 03:01 and DRB1 ${ }^{*} 04: 01$, we reanalyzed the data excluding $\mathrm{DRB1}^{*}$ 03:01/04:01 probands, representing a significant reduction in the sample size (see Table 3 ). The $64 \mathrm{cM}$ linkage signal remained prominent in this subset with no evidence for heterogeneity (LOD $=2.0$ and HLOD = 2.2), despite the reduced sample size. In contrast, the peak located at $42 \mathrm{cM}$ decreased substantially with stratification: LOD $=0.9$ and HLOD $=1.6$ (unstratified: $\mathrm{LOD}=3.6$ and $\mathrm{HLOD}=5.0$ ), supporting the "protective" effect of DRB1" 03:01 on RET.

3.4.3. DRB1 04:01 Stratification (DRB1 $\left.1^{*} 04: 01 / X\right)$, (Figure 2(c)). We analyzed the RET data using only families of probands carrying the $\mathrm{DRB1}^{*} 04: 01$ allele, including $\mathrm{DRB1}^{*}$ 03:03/DRB1 ${ }^{*}$ 04:01 heterozygotes. The LOD and HLOD scores at the $42 \mathrm{cM}$ locus in the DRB1 ${ }^{*} 04$ :01-stratified analysis remained high (4.1 and 4.1, resp.). This suggests that heterogeneous loci contributing to RET were eliminated in the stratified sample (hence the increase in the LOD score) but some families contributing to the LOD score were also eliminated (thus the decrease in the HLOD). At the $64 \mathrm{cM}$ locus, the HLOD decreased (HLOD $=0.9$ ) compared to the unstratified analysis ( HLOD = 2.2); the LOD scores remained negative (unstratified $=-1.5$ and stratified $=-0.5$ ). This is in sharp contrast to the DRB1 ${ }^{*}$ 03:01 stratification findings, in which the evidence for linkage at the $64 \mathrm{cM}$ locus was notably stronger than in the unstratified analysis. This suggests that the $64 \mathrm{cM}$ locus interacts with the $\mathrm{DRB1}^{*}$ 03:01 allele to foster the expression of RET but that it does not interact with the $\mathrm{DRB1}^{*}$ 04:01 allele. This conclusion is strengthened when we look at the results of the "pure" DRB1 ${ }^{*}$ 04:01 family analysis (see below).

3.4.4. "Pure" DRB1 04:01 Stratification (DRB1 04:01/X, (X\# 03:01)), (Figure $2(d)$ ). When including only $\mathrm{DRB1}^{*} 04: 01 / \mathrm{X}$, ( $\mathrm{X} \neq 03: 01)$ families, the signal at the $42 \mathrm{cM}$ location remains notable with no evidence of heterogeneity (LOD $=2.5$ and HLOD = 2.5), despite the large drop in sample size (see Table 3 ). At the $64 \mathrm{cM}$ locus, the evidence is against linkage $(\mathrm{LOD}=-1.0$ and HLOD $=0.0)$.

The stratification analysis results suggest that the $42 \mathrm{cM}$ and the $64 \mathrm{cM}$ loci interact epistatically and differentially with the DRB1 ${ }^{*}$ 03:01 and * 04:01 alleles, revealing evidence of locus heterogeneity for each phenotype. The unstratified analysis of the RET families at the $42 \mathrm{cM}$ locus yields a LOD score of 3.6 and an HLOD of 5.0, indicating substantial locus heterogeneity in the data. Analysis of "pure" 03:01 families 
yields a LOD $<1$ for RET at the $42 \mathrm{cM}$ locus and an HLOD that still suggests heterogeneity $(\mathrm{HLOD}=1.6)$. The "pure" 04:01 families yield a $\mathrm{LOD}=\mathrm{HLOD}=2.5$ at the $42 \mathrm{cM}$ locus. These results suggest that the $42 \mathrm{cM}$ locus interacts positively with the 04:01 allele (to produce RET) and negatively with the 03:01 allele (to protect against RET). Furthermore, when only the 04:01 allele is present (and not 03:01), the $42 \mathrm{cM}$ locus shows no evidence of heterogeneity.

The DRB1 ${ }^{*} 03: 01$ stratification analyses suggest that the $42 \mathrm{cM}$ locus influences RET less when ${ }^{*}$ 03:01 is present than when *04:01 is present. This is expected if *03:01 "protects" against RET. Like the $42 \mathrm{cM}$ locus, the $64 \mathrm{cM}$ locus shows evidence of interaction, but with the *03:01 allele. When stratifying on the $04: 01$ allele, there is almost no evidence for linkage at the $64 \mathrm{cM}$ locus.

\section{Discussion}

In this study, we used, for the first time, LOD score linkage analysis to identify loci that contribute to the expression of the microvascular complications of RET and NEPH. Linkage analysis has been shown to have the most power to detect loci important for disease expression and has the greatest ability to give us information about the genetic characteristics of the phenotype and the existence of heterogeneity [22].

The results of the "any complication" phenotype indicated the existence of three loci. The fact that the HLA locus appeared is unsurprising $[10,26-31]$ but the discovery of two novel, complications-related loci at $42 \mathrm{cM}$ and at $64 \mathrm{cM}$ was unexpected. There was also significant evidence for the interaction of these two novel loci with the alleles DRB1 ${ }^{*}$ 03:01 and ${ }^{*} 04: 01$.

Statistically significant evidence for the existence of two loci for complications adds strong support for inherited influences on complications' expression. The two loci appear to affect RET and NEPH differently. Both appeared to influence RET expression but the $64 \mathrm{cM}$ locus appeared to influence only NEPH and the $42 \mathrm{cM}$ locus had no influence on NEPH.

The significant change in the $64 \mathrm{cM}$ locus's LOD score among proband families with DRB1* 03:01 strongly suggests that the presence of DRB1 ${ }^{*}$ 03:01 increases the influence of the $64 \mathrm{cM}$ locus on RET expression. The influence of the $64 \mathrm{cM}$ locus virtually disappears when the proband has the 04:01 allele while the $42 \mathrm{cM}$ peak is strengthened. When proband families are not selected for having a particular HLA allele, the observed evidence for heterogeneity is expected if the two HLA alleles contribute differentially to the phenotype. The positive differences in the LOD scores between the stratified and unstratified samples are strong indicators of interaction of the HLA alleles with the two loci [32].

The strong linkage evidence at the HLA region $(52 \mathrm{cM})$ might indicate the influence of HLA on complications (known from association evidence) or merely cosegregation of HLA alleles with diabetes in general regardless of complications. Changes in the HLA linkage profile under stratification are not interpretable because we artificially altered the HLA allele structure by including or excluding specific alleles. However, finding that the DRB1 ${ }^{*} 03: 01$ and
* 04:01 alleles interact with the novel loci confirms that HLA influences complications' expression.

This study is not without ambiguities.

(1) The linkage region we have identified $(30 \mathrm{cM}-70 \mathrm{cM})$ is a relatively small one for most linkage analyses; yet we have observed three distinct loci with specific effects. One of those loci is the HLA region, which strongly affects T1D expression. Were we analyzing the T1D phenotype and not complications, the LOD score at HLA would be on the order of 40 , thus swamping any other T1D-related signals. However, the narrowness of the region does not nullify the clear separateness of the linkage signals. No matter how the data are stratified and broken down, the consistency of the $42 \mathrm{cM}$ and $64 \mathrm{cM}$ peaks, even when the $42 \mathrm{cM}$ peak disappears (as in the analysis of NEPH), argues strongly that these loci influence complications' expression.

(2) The number of linkage analyses that we have performed may lead to the question as to whether the LOD scores for the two loci we identified should be subject to correction for genome-wide testing. All three peaks appear in the first analysis with notable (2.5-5) LOD scores and/or HLOD scores and the locations of these peaks were invariant. The information content of the genotypic data did not fall below $98 \%$ across the region. The usual criterion for significance of a LOD score (variously debated to be from 2.5 to 4.0 ) is for genome-wide significance, that is, examining marker loci over the entire genome. However, we examined only markers on chromosome 6 , which constitutes only about $6 \%$ of the genome and, therefore, the genome-wide cut-off values are too conservative for this analysis. Even so, several of the peaks did reach genome-wide significance levels, which became even higher under stratification (i.e., with a smaller dataset). We did not attempt to correct LOD scores under stratification because the stratification hypothesis did not concern the existence of a peak but how it changed under stratification. Some of those changes would have to be viewed as statistically significant indications of interaction [32].

(3) We used changes in the height of the peaks as indicators of the loci's influence on complications' expression. The question of the relative strengths of influence on gene expression as related to linkage peak height is not a well-studied area. Linkage will most likely only be observed with loci "necessary" for disease expression [33]. Using the relative LOD score changes as indicators of heterogeneity and of interaction is an expansive use of linkage analysis that has been applied to good effect in our studies of autoimmune thyroid disease [34] as well as other diseases [35]. The information inherent in linkage analysis is extremely rich and can be exploited to learn about heterogeneity, mode of inheritance, pleiotropy, and gene-gene interaction. 
(4) Previous work has demonstrated how the stratification technique we used can identify epistatically interacting loci [25]. Recent work [32] on detecting interaction has shown that epistasis is easily differentiated from heterogeneity and that false positive indications of interaction are unlikely. However, we cannot yet quantify the degree of interaction based on changes in the LOD score. Also, because of sample size changes, changes in LOD scores cannot always be unambiguously assigned to changes in interaction. However, an increase in the LOD score that accompanies a decrease in the sample size argues for a "purification" of the sample. Thus, an increase based on stratification is a clear indicator of interaction.

The next step in this work is to analyze the whole genome, now that we know the importance of stratification loci in identifying interaction. While applying this study's stratification approach may help us identify the specific genes in the linkage regions using association analysis of SNPs with retinopathy and/or nephropathy, the option also now exists to use next-generation sequencing to identify the disease-related variants. The difficulty, as with other common conditions, is identifying the disease-related variant if such variants do not occur in exons.

Replicating our work in other samples is highly desirable. However, since the wide adoption of GWAS as the genetic technique of choice and the accompanying decrease in the collection of family data, it is not clear how much family data exist for linkage of complications. Nevertheless, family studies are the best way to effectively use the newest genetic technologies [36], and we hope that our findings will inspire a resurgence of family studies for T1D complications and the search for heterogeneity.

\section{Conflict of Interests}

The authors declare that there is no conflict of interests associated with this paper.

\section{Authors' Contribution}

Ettie M. Lipner chose and implemented the statistical analyses and wrote the paper. Yaron Tomer contributed ideas and suggested analyses and reviewed the paper. Janelle A. Noble generated the HLA data and contributed valuable information to the paper. Maria C. Monti cleaned the data and did the initial familial analyses and reviewed the paper. John T. Lonsdale provided the data and supervised the updating of the clinical information. Barbara Corso did genetic data cleaning and database quality control. David A. Greenberg initiated the study, contributed analysis ideas, supervised the analyses, and contributed to the paper.

\section{Acknowledgments}

The authors thank Rebecca Yohannes (Columbia University), Junying Zhang (Columbia University), Ryan Subaran (Nationwide Children's Hospital), and Abbie Neininger
(Nationwide Children's Hospital) for programming and analysis assistance. This project is funded, in part, under a grant with the Pennsylvania Department of Health. The Department specifically disclaims responsibility for any analyses, interpretations, or conclusions (John T. Lonsdale). This research was also supported in part by Grants 2T15 LM009451 (Ettie M. Lipner); NINDS NS27941, NIMH MH48858, NS61829, NS70323, Nationwide Children's Hospital, and the New York State Psychiatric Institute (David A. Greenberg); DK61722 (Janelle A. Noble); DK61659, DK067555, and DK073681 (Yaron Tomer).

\section{References}

[1] M. C. Monti, J. T. Lonsdale, C. Montomoli, R. Montross, E. Schlag, and D. A. Greenberg, "Familial risk factors for microvascular complications and differential male-female risk in a large cohort of American families with type 1 diabetes," Journal of Clinical Endocrinology and Metabolism, vol. 92, no. 12, pp. 4650-4655, 2007.

[2] V. Harjutsalo, Familial Aggregation of Type 1 Diabetes and Diabetic Nephropathy in Finland, National Public Health Institute, 2007.

[3] M. Quinn, M. C. Angelico, J. H. Warram, and A. S. Krolewski, "Familial factors determine the development of diabetic nephropathy in patients with IDDM," Diabetologia, vol. 39, no. 8, pp. 940-945, 1996.

[4] E. R. Seaquist, F. C. Goetz, S. Rich, and J. Barbosa, "Familial clustering of diabetic kidney disease. Evidence for genetic susceptibility to diabetic nephropathy," The New England Journal of Medicine, vol. 320, no. 18, pp. 1161-1165, 1989.

[5] A.-M. Österholm, B. He, J. Pitkaniemi et al., "Genome-wide scan for type 1 diabetic nephropathy in the Finnish population reveals suggestive linkage to a single locus on chromosome 3q," Kidney International, vol. 71, no. 2, pp. 140-145, 2007.

[6] R. P. Igo Jr., S. K. Iyengar, S. B. Nicholas et al., "Genomewide linkage scan for diabetic renal failure and albuminuria: the find study," American Journal of Nephrology, vol. 33, no. 5, pp. 381389, 2011.

[7] S. K. Iyengar, H. E. Abboud, K. A. B. Goddard et al., "Genomewide scans for diabetic nephropathy and albuminuria in multiethnic populations: the family investigation of nephropathy and diabetes (FIND)," Diabetes, vol. 56, no. 6, pp. 1577-1585, 2007.

[8] D. K. Moczulski, J. J. Rogus, A. Antonellis, J. H. Warram, and A. S. Krolewski, "Major susceptibility locus for nephropathy in 1 diabetes on chromosome 3q: results of novel discordant sibpair analysis," Diabetes, vol. 47, no. 7, pp. 1164-1169, 1998.

[9] S. K. Iyengar, K. A. Fox, M. Schachere et al., "Linkage analysis of candidate loci for end-stage renal disease due to diabetic nephropathy," Journal of the American Society of Nephrology, vol. 14, pp. S195-S201, 2003.

[10] E. M. Lipner, Y. Tomer, J. A. Noble et al., "HLA class I and II alleles are associated with microvascular complications of type 1 diabetes," Human Immunology, vol. 74, no. 5, pp. 538-544, 2013.

[11] A. J. M. Boulton, A. I. Vinik, J. C. Arezzo et al., "Diabetic neuropathies: a statement by the American Diabetes Association," Diabetes Care, vol. 28, no. 4, pp. 956-962, 2005.

[12] Expert Committee on the Diagnosis and Classification of Diabetes Mellitus, "Report of the expert committee on the diagnosis and classification of diabetes mellitus," Diabetes Care, vol. 26, supplement 1, pp. S5-S20, 2003. 
[13] D. S. Fong, L. Aiello, T. W. Gardner et al., "Diabetic retinopathy," Diabetes Care, vol. 26, supplement 1, pp. S99-S102, 2003.

[14] M. E. Molitch, R. A. DeFronzo, M. J. Franz et al., "Diabetic nephropathy," Diabetes Care, vol. 26, supplement 1, pp. S94-S98, 2003.

[15] C. Tiberti, R. Buzzetti, E. Anastasi et al., "Autoantibody negative new onset Type 1 diabetic patients lacking high risk HLA alleles in a Caucasian population: are these Type $1 \mathrm{~b}$ diabetes cases?" Diabetes/Metabolism Research and Reviews, vol. 16, no. 1, pp. 8$14,2000$.

[16] S. Hameed, S. Ellard, H. J. Woodhead et al., "Persistently autoantibody negative (PAN) type 1 diabetes mellitus in children," Pediatric Diabetes, vol. 12, no. 3, pp. 142-149, 2011.

[17] S. S. Merkin, K. Cavanaugh, J. C. Longenecker, N. E. Fink, A. S. Levey, and N. R. Powe, "Agreement of self-reported comorbid conditions with medical and physician reports varied by disease among end-stage renal disease patients," Journal of Clinical Epidemiology, vol. 60, no. 6, pp. 634-642, 2007.

[18] J. R. Robinson, T. K. Young, L. L. Roos, and D. E. Gelskey, "Estimating the burden of disease: comparing administrative data and self-reports," Medical Care, vol. 35, no. 9, pp. 932-947, 1997.

[19] D. M. W. Kriegsman, B. W. J. H. Penninx, J. T. M. Van Eijk, A. J. P. Boeke, and D. J. H. Deeg, "Self-reports and general practitioner information on the presence of chronic diseases in community dwelling elderly. A study on the accuracy of patients' selfreports and on determinants of inaccuracy," Journal of Clinical Epidemiology, vol. 49, no. 12, pp. 1407-1417, 1996.

[20] D. A. Greenberg, "There is more than one way to collect data for linkage analysis: what a study of epilepsy can tell us about linkage strategy for psychiatric disease," Archives of General Psychiatry, vol. 49, no. 9, pp. 745-750, 1992.

[21] L. Kruglyak, M. J. Daly, M. P. Reeve-Daly, and E. S. Lander, "Parametric and nonparametric linkage analysis: a unified multipoint approach," American Journal of Human Genetics, vol. 58, no. 6, pp. 1347-1363, 1996.

[22] D. A. Greenberg and P. C. Abreu, "Determining trait locus position from multipoint analysis: accuracy and power of three different statistics," Genetic Epidemiology, vol. 21, no. 4, pp. 299314, 2001.

[23] S. E. Hodge, P. C. Abreu, and D. A. Greenberg, "Magnitude of type I error when single-locus linkage analysis is maximized over models: a simulation study," The American Journal of Human Genetics, vol. 60, no. 1, pp. 217-227, 1997.

[24] D. A. Greenberg, P. Abreu, and S. E. Hodge, "The power to detect linkage in complex disease by means of simple LODscore analyses," The American Journal of Human Genetics, vol. 63, no. 3, pp. 870-879, 1998.

[25] L. Rodriguez-Murillo, R. Subaran, W. C. L. Stewart et al., "Novel loci interacting epistatically with bone morphogenetic protein receptor 2 cause familial pulmonary arterial hypertension," Journal of Heart and Lung Transplantation, vol. 29, no. 2, pp. 174-180, 2010.

[26] S. K. Cordovado, Y. Zhao, J. H. Warram et al., "Nephropathy in type 1 diabetes is diminished in carriers of HLA-DRB1* 04: the genetics of kidneys in diabetes (GoKinD) study," Diabetes, vol. 57, no. 2, pp. 518-522, 2008.

[27] D. Agardh, L. K. Gaur, E. Agardh, M. Landin-Olsson, C.-D. Agardh, and Å. Lernmark, "HLA-DQB1*0201/0302 is associated with severe retinopathy in patients with IDDM," Diabetologia, vol. 39, no. 11, pp. 1313-1317, 1996.
[28] T. Mimura, H. Funatsu, Y. Uchigata et al., "Relationship between human leukocyte antigen status and proliferative diabetic retinopathy in patients with younger-onset type 1 diabetes mellitus," American Journal of Ophthalmology, vol. 135, no. 6, pp. 844-848, 2003.

[29] R. A. Jensen, E. Agardh, Å. Lernmark et al., "HLA genes, islet autoantibodies and residual C-peptide at the clinical onset of type 1 diabetes mellitus and the risk of retinopathy 15 years later," PLoS ONE, vol. 6, no. 3, Article ID e17569, 2011.

[30] A. Svejgaard, B. K. Jakobsen, P. Platz et al., "HLA associations in insulin-dependent diabetes: search for heterogeneity in different groups of patients from a homogeneous population," Tissue Antigens, vol. 28, no. 4, pp. 237-244, 1986.

[31] K. J. Cruickshanks, C. M. Vadheim, S. E. Moss et al., "Genetic marker associations with proliferative retinopathy in persons diagnosed with diabetes before $30 \mathrm{yr}$ of age," Diabetes, vol. 41, no. 7, pp. 879-885, 1992.

[32] B. Corso and D. A. Greenberg, "Using linkage analysis to detect gene-gene interaction by stratifying family data on known disease, or disease-associated, alleles," PLoS ONE, vol. 9, no. 4, Article ID e93398, 2014.

[33] D. A. Greenberg, "Linkage analysis of 'necessary' disease loci versus 'susceptibility' loci," The American Journal of Human Genetics, vol. 52, no. 1, pp. 135-143, 1993.

[34] V. J. Vieland, Y. Huang, C. Bartlett, T. F. Davies, and Y. Tomer, "A multilocus model of the genetic architecture of autoimmune thyroid disorder, with clinical implications," The American Journal of Human Genetics, vol. 82, no. 6, pp. 1349-1356, 2008.

[35] M. Durner, M. A. Keddache, L. Tomasini et al., "Genome scan of idiopathic generalized epilepsy: evidence for major susceptibility gene and modifying genes influencing the seizure type," Annals of Neurology, vol. 49, no. 3, pp. 328-335, 2001.

[36] D. A. Greenberg and W. C. L. Stewart, "How should we be searching for genes for common epilepsy? A critique and a prescription," Epilepsia, vol. 53, supplement 4, pp. 72-80, 2012. 


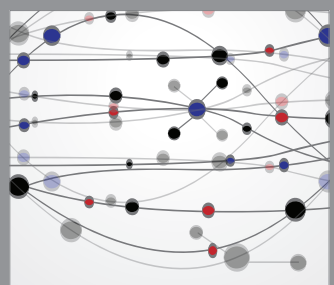

The Scientific World Journal
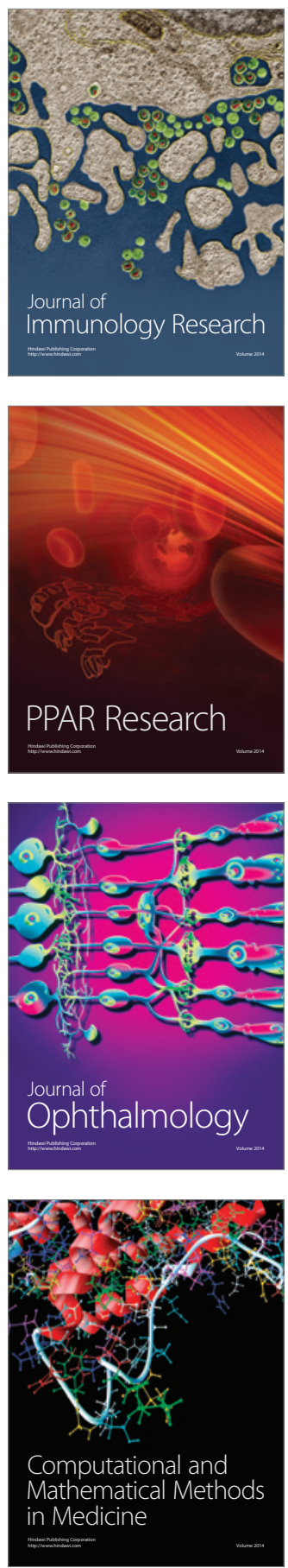

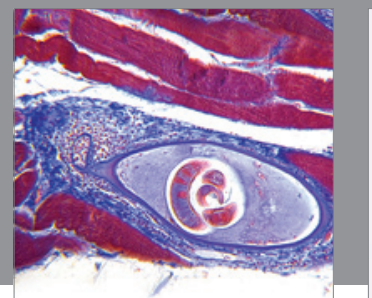

Gastroenterology

Research and Practice
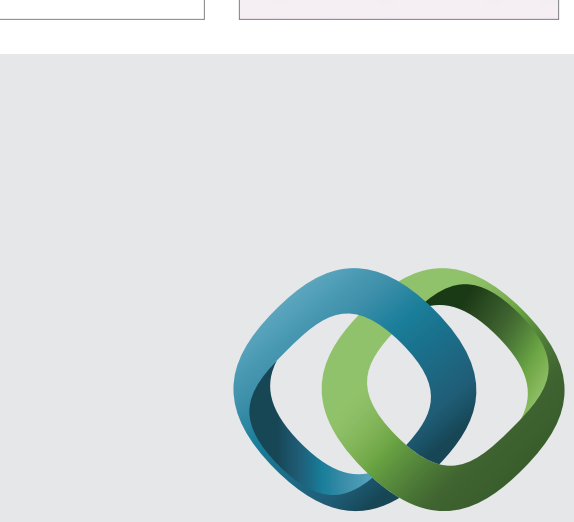

\section{Hindawi}

Submit your manuscripts at

http://www.hindawi.com
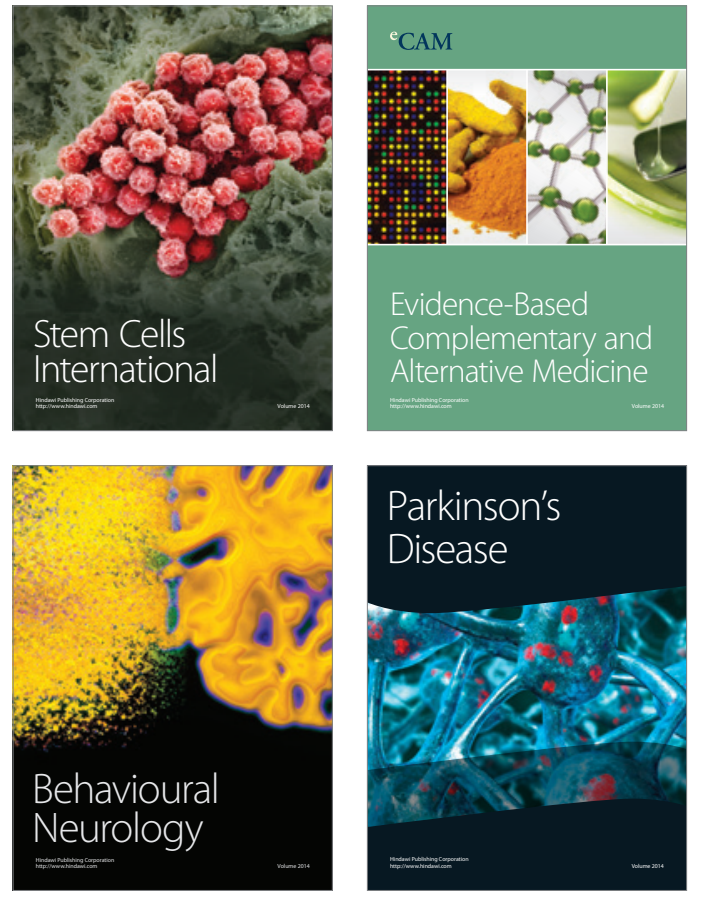
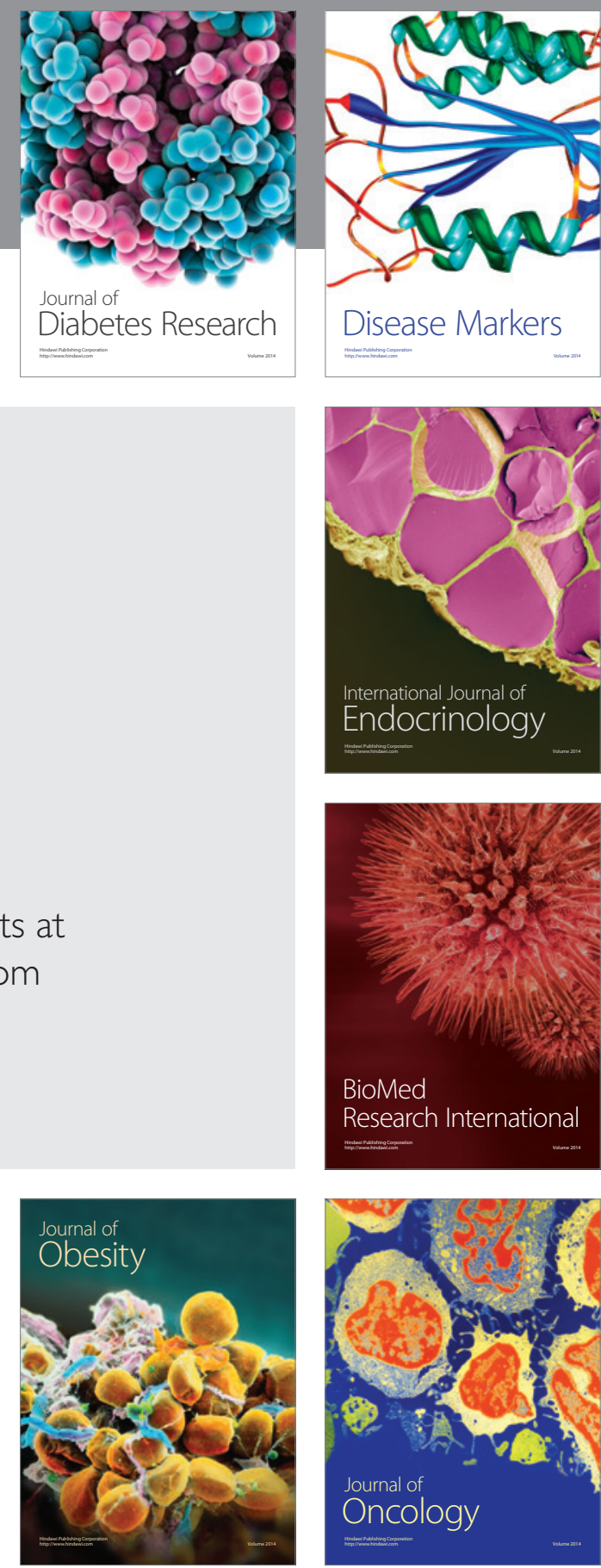

Disease Markers
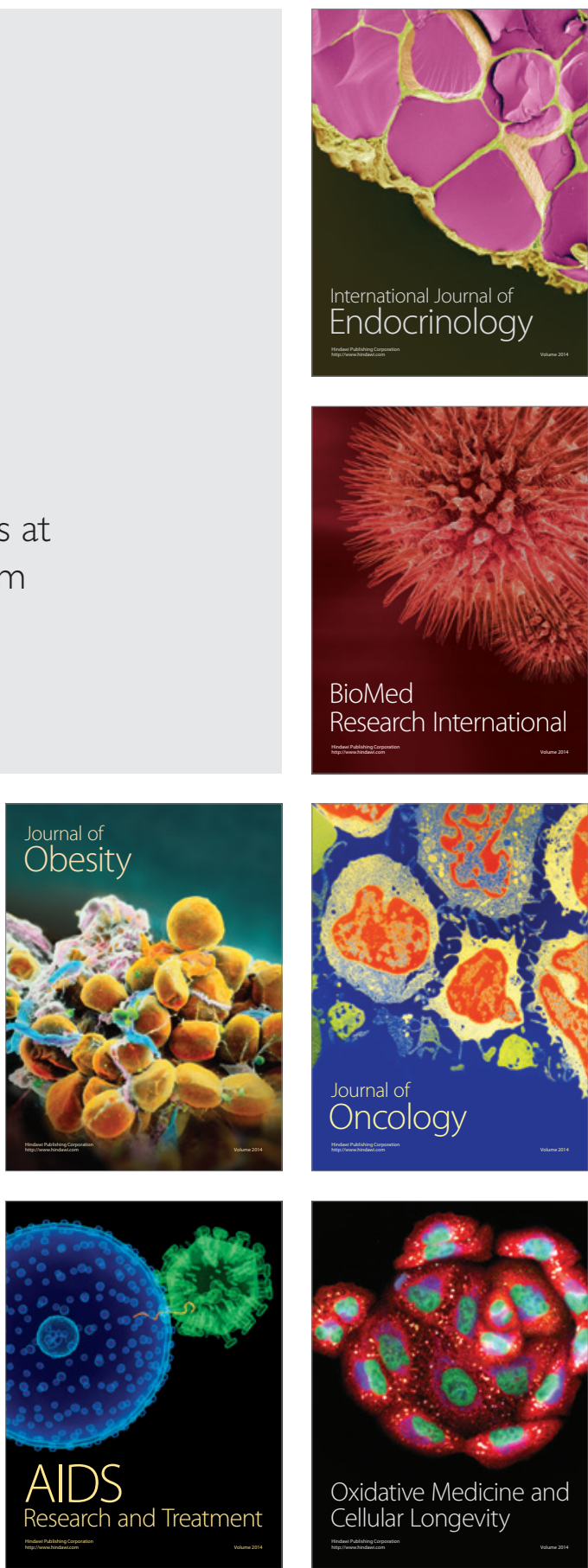